\section{Epidemiologie lebensbedrohlicher Anaphylaxien}

\author{
Valide Daten zur Inzidenz schwerer anaphylaktischer Reaktionen \\ sind rar. Einen Fortschritt wird in absehbarer Zeit die konsequente \\ Anwendung des neuen ICD-10-Schlüssels bringen, der auch den \\ Auslöser einer Anaphylaxie kodiert. Den aktuellen Status quo fasst \\ eine Übersicht aus Frankreich zusammen.
}

$\mathrm{D}$ ie bisher verfügbaren epidemiologischen Daten zu anaphylaktischen Reaktionen basieren auf Fragebogenerhebungen. Dazu wurden verschiedene nationale und regionale Probandenkollektive erfasst, indem Notfallambulanzen und Krankenhäuser befragt wurden oder Adrenalinverordnungen gezählt wurden. Aus den Ergebnissen verschiedener Arbeitsgruppen lässt sich abschätzen, dass etwa $0,4 \%$ der Fälle in einer Notaufnahme durch eine Allergie bedingt sind und etwa $0,04 \%$ schwere Anaphylaxien sind. Die Inzidenz einer schweren Anaphylaxie in der Durchschnittsbevölkerung wird auf ein bis drei
Personen pro 10.000, die Inzidenz einer letalen Anaphylaxie auf ein bis drei Personen pro 1.000 .000 geschätzt.

Auslösende Allergene sind in den meisten Fällen Nahrungsmittel, Hymenopterenstiche und Medikamente. Unter den Nahrungsmitteln spielen vor allem Erdnüsse, andere Leguminosen, Gemüsesorten, Krustazeen sowie Weizen, Milch und Sellerie eine Rolle. Bei den Hymenopteren sind es vor allem die Stiche von Wespen, Bienen und Hornissen, nach denen schwere Anaphylaxien auftreten. Bei den durch Medikamente ausgelösten Anaphylaxien waren zu über 50\% Antibiotika die verantwortlichen Allergene, vor allem Penicilline und Cephalosporine, ferner nicht steroidale Antirheumatika sowie Latex. Eine gefährliche Eigenart der Medikamentenallergie ist das schnelle und heftige Auftreten der klinischen Symptomatik oft schon Minuten nach der Applikation und unabhängig vom Applikationsweg. Bei Einleitung oder während einer Narkose waren hauptsächlich Muskelrelaxanzien, aber auch Latex und Antibiotika die auslösenden Allergene.

Wichtig ist, dass nach erfolgreicher Notfallbehandlung eine eingehende allergologische Untersuchung mit der Identifikation des auslösenden Allergens erfolgt und das Ergebnis dokumentiert wird. Hierdurch werden in Zukunft die verantwortlichen Allergene differenzierter als bisher erfasst und auch regionale Besonderheiten erkennbar werden.

Dr. Dieter Bruchhausen, Wuppertal

\section{Literatur}

Moneret-Vautrin DA, Morisset M, Flabbee J, Beaudouin E, Kanny G. Epidemiology of lifethreatening and lethal anaphylaxis: a review. Allergy 2005; 60: 443-51

\title{
Beruflich bedingte Hautkrankheiten bei Musikern
}

\section{Allergische Hauterkrankungen manifestieren sich bei Musikern ganz überwiegend als Kontaktdermatitiden an Mund und Händen.} Welche Allergene dabei eine Rolle spielen, hat eine italienische Studie untersucht.

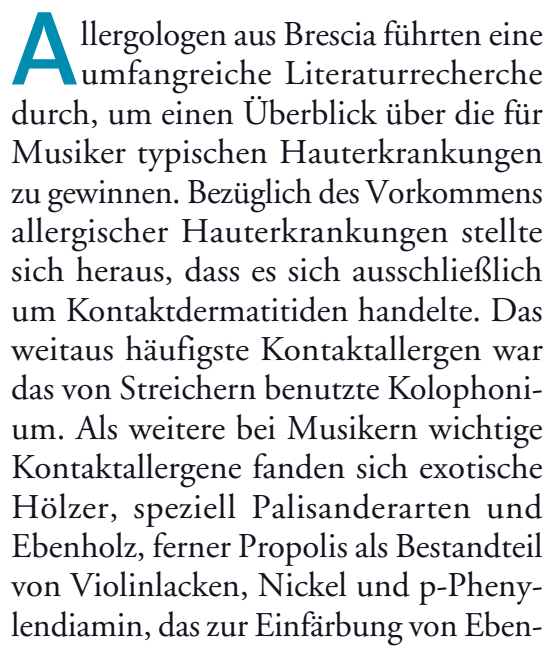

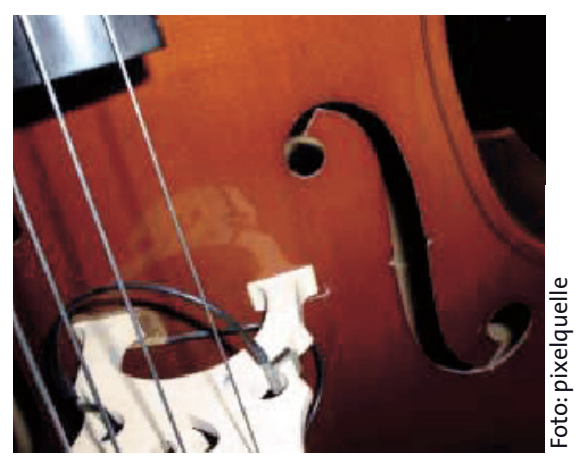

Der häufigste Auslöser von Kontaktallergien bei Berufsmusikern ist das Kolophonium, das von Streichern dazu benutzt wird, die Bogenbespannung für die Saiten adhärenter zu machen. holz verwendet wird. Die Ekzeme sind an den für das jeweilige Instrument typischen Kontaktstellen lokalisiert.

Der untersuchende Allergologe sollte sich vom Musiker dessen Instrument und seine Handhabung vorführen lassen, um die Ekzemstelle einem Instrumentenkontakt zuordnen zu können. Möglicherweise ergeben sich so auch schon Hinweise auf die Art des Kontaktallergens. Falls Testallergene kommerziell nicht erhältlich sind, kann versucht werden, native Testsubstanzen aus den jeweiligen Instrumentenwerkstätten zu besorgen.

Die schwierige Frage, wie eine Allergendistanzierung zu erreichen ist, kann nur jeweils für den Individualfall gelöst werden. Oftmals wäre die komplette Meidung des Instruments die einzige Lösung, was aber einer Berufsaufgabe gleichkommt.

\section{Dr. Dieter Bruchhausen, Wuppertall}

\section{Literatur}

Lombardi C, Bottello M, Caruso A, Gargioni S, Passalacqua G. Allergy and skin diseases in musicians. Allerg Immunol (Paris) 2003; 35: 52-5 